\title{
Random products of automorphisms of Heisenberg nilmanifolds and Weil's representation
}

\author{
Bachir Bekka and Jean-Romain Heu
}

November 1, 2009

\begin{abstract}
For $n \geq 1$, let $H$ be the $(2 n+1)$-dimensional real Heisenberg group, and let $\Lambda$ be a lattice in $H$. Let $\Gamma$ be a group of automorphisms of the corresponding nilmanifold $\Lambda \backslash H$ and $U$ the associated unitary representation of $\Gamma$ on $L^{2}(\Lambda \backslash H)$. Denote by $T$ the maximal torus factor associated to $\Lambda \backslash H$. Using Weil's representation (also known as the metaplectic representation), we show that a dense set of matrix coefficients of the restriction of $U$ to the orthogonal complement of $L^{2}(T)$ in $L^{2}(\Lambda \backslash H)$ belong to $\ell^{4 n+2+\varepsilon}(\Gamma)$ for every $\varepsilon>0$.

We give the following application to random walks on $\Lambda \backslash H$ defined by a probability measure $\mu$ on $\operatorname{Aut}(\Lambda \backslash H)$. Denoting by $\Gamma$ the subgroup of $\operatorname{Aut}(\Lambda \backslash H)$ generated by the support of $\mu$ and by $U^{0}$ and $V^{0}$ the restrictions of $U$ respectively to the subspaces of $L^{2}(\Lambda \backslash H)$ and $L^{2}(T)$ with zero mean, we prove the following inequality:

$$
\left\|U^{0}(\mu)\right\| \leq \max \left\{\left\|V^{0}(\mu)\right\|,\left\|\lambda_{\Gamma}(\mu)\right\|^{1 /(2 n+2)}\right\},
$$

where $\lambda_{\Gamma}$ is the left regular representation of $\Gamma$ on $\ell^{2}(\Gamma)$. In particular, the action of $\Gamma$ on $\Lambda \backslash H$ has a spectral gap if and only if the corresponding action of $\Gamma$ on $T$ has a spectral gap.
\end{abstract}

\section{Introduction}

Let $(X, m)$ be a probability space and $G$ a locally compact group of measure preserving transformations of $X$. Given a probability measure $\mu$ on $G$, 
consider a sequence of independent $\mu$-distributed random variables $X_{n}^{\omega}$ with values in $G$ and the corresponding random products $S_{n}^{\omega}=X_{n}^{\omega} \ldots X_{1}^{\omega}$ for $n \in \mathbf{N}$. This defines a random walk on $X$ with initial distribution $m$ and trajectories $S_{n}^{\omega}(x)$ for $x \in X$ and $n \in \mathbf{N}$. A question of interest is whether this random walk has a spectral gap. To define this notion, let $U$ be the unitary representation of $G$ on $L^{2}(X, m)$ defined by $U_{g}(\xi)=\xi\left(g^{-1}(x)\right)$ for $g \in$ $G, \xi \in L^{2}(X, m)$, and $x \in X$. Let $L_{0}^{2}(X, m)$ be the $U(G)$-invariant subspace of functions $\xi$ in $L^{2}(X, m)$ with zero mean, that is, with $\int_{X} \xi(x) d m(x)=0$. Denote by $U^{0}$ the restriction of $U$ to $L_{0}^{2}(X, m)$. Let $U^{0}(\mu)$ be the convolution operator defined on $L_{0}^{2}(X, m)$ by

$$
U^{0}(\mu) \xi=\int_{G} U_{g}^{0}(\xi) d \mu(g) \quad \text { for all } \quad \xi \in L_{0}^{2}(X, m) .
$$

Observe that $\left\|U^{0}(\mu)\right\| \leq 1$. We say that $\mu$ has a spectral gap in $(X, m)$ if $\left\|U^{0}(\mu)\right\|<1$. This spectral gap property has several interesting applications; the most immediate one is the exponentially fast convergence of the sequence of functions $x \mapsto \mathbf{E}\left(\xi\left(S_{n}^{\omega}(x)\right)\right.$ to $\int_{X} \xi d m$ in the $L^{2}$-norm for every $\xi \in L^{2}(X, m)$. Other applications include the existence of a rate of convergence for random ergodic theorems, a central limit theorem, and the uniqueness of invariant means on $L^{\infty}(X, m)$; see [FuSh99], [Guiv05], [Lubo94], [Sarn90].

The spectral gap property can be formulated in terms of weak containment of group representations (see [BeHV08, G.4.2]). Assume that the subgroup generated by the support of $\mu$ is dense in $G$. Assume moreover that $\mu$ is aperiodic (that is, the support of $\mu$ is not contained in the coset of a proper closed subgroup of $G$ ). Then $\mu$ has a spectral gap in $(X, m)$ if and only if there is no $G$-almost invariant vectors in $L_{0}^{2}(X, m)$. If this is the case, we say for short that the $G$-action on $X$ has a spectral gap.

We emphasize that the existence of a spectral gap property is a phenomenon which can occur only in the context of non-amenable groups: when $G$ is a discrete amenable group and $m$ is non-atomic, then $G$ has never a spectral gap on $X$ (see [JuRo79] or [Schmi80]).

When $X$ is the $n$-dimensional torus $\mathbf{R}^{n} / \mathbf{Z}^{n}$, equipped with the normalized Lebesgue measure $m$, sufficient conditions were given in [FuSh99] for the existence of a spectral gap for the action of a subgroup of $G L_{n}(\mathbf{Z})$ by automorphisms on $\mathbf{R}^{n} / \mathbf{Z}^{n}$ (see also Example 4 below). In this paper, we will consider the case where $X$ is a Heisenberg nilmanifold and $G$ a group of automorphisms of $X$. 
For $n \geq 1$, let $H=H_{2 n+1}(\mathbf{R})$ be the $(2 n+1)$-dimensional real Heisenberg group. This is a two step nilpotent Lie group with one-dimensional centre $Z$ (see Section 2 below). Let $\Lambda$ be a lattice in $H: \Lambda$ is a discrete subgroup of $H$ such that there exists a (unique) probability measure $m$ on the Borel sets of the corresponding nilmanifold $\Lambda \backslash H$ which is invariant under right translation by elements from $H$. (Observe that $\Lambda$ is cocompact in $H$.) Denote by $\operatorname{Aut}(H)$ the group of continuous automorphisms of $H$ and by $\operatorname{Aut}(\Lambda \backslash H)$ the subgroup of all $g \in \operatorname{Aut}(H)$ such that $g(\Lambda)=\Lambda$; every automorphism $g \in \operatorname{Aut}(\Lambda \backslash H)$ induces a homeomorphism of $\Lambda \backslash H$.

Let $\Gamma$ be a subgroup of $\operatorname{Aut}(\Lambda \backslash H)$. The action of $\Gamma$ on $\Lambda \backslash H$ preserves the $H$-invariant probability measure $m$ on $\Lambda \backslash H$. Let $U$ be the associated unitary representation of $\Gamma$ on $L^{2}(\Lambda \backslash H, m)$. Let $T=\Lambda Z \backslash H$ be the maximal torus factor of $\Lambda \backslash H$. Observe that $T \cong \mathbf{R}^{2 n} / \mathbf{Z}^{2 n}$ and $\operatorname{Aut}(T) \cong G L_{2 n}(\mathbf{Z})$. Since $\operatorname{Aut}(\Lambda \backslash H)$ preserves $Z \Lambda$, we have a homomorphism $p: \operatorname{Aut}(\Lambda \backslash H) \rightarrow \operatorname{Aut}(T)$ and an induced action of $\Gamma$ on $T$. This defines a unitary representation of $\Gamma$ on $L^{2}(T)$, where $T$ is equipped with normalized Lebesgue measure. We can (and will) identify $L^{2}(T)$, as $\Gamma$-space, with a closed $U(\Gamma)$-invariant subspace of $L^{2}(\Lambda \backslash H)$. Denote by $\mathcal{H}$ the orthogonal complement of $L^{2}(T)$ in $L^{2}(\Lambda \backslash H)$, so that we have an orthogonal decomposition

$$
L^{2}(\Lambda \backslash H)=L^{2}(T) \oplus \mathcal{H}
$$

into $U(\Gamma)$-invariant subspaces. Here is our main result.

Theorem 1 The matrix coefficients of the restriction of $U$ to $\mathcal{H}$ are strongly $L^{4 n+2+\varepsilon}$ : there are dense subspaces $D_{1}$ and $D_{2}$ of $\mathcal{H}$ such that, for any $v \in D_{1}$ and $w \in D_{2}$, the matrix coefficient $\gamma \mapsto\left\langle U_{\gamma} v, w\right\rangle$ belongs to $\ell^{4 n+2+\varepsilon}(\Gamma)$, for every $\varepsilon>0$.

Concerning the proof of the previous theorem, we first show that the representation $U$ is linked with Weil's representation, which is also known as Segal-Shale-Weil, metaplectic, or oscillator representation (see [Shal62], [Weil64]). The crucial tool is then a result from [HoMo79] about the decay of the matrix coefficients of Weil's representation.

Here is an immediate consequence of Theorem 1. Recall that, if $X$ is a locally compact space, $C_{0}(X)$ denotes the space of complex-valued continuous functions on $X$ which tend to zero at infinity.

Corollary 2 The restriction of the unitary representation $U$ to $\mathcal{H}$ is mixing: the matrix coefficients $\gamma \mapsto\left\langle U_{\gamma} v, w\right\rangle$ belong to $c_{0}(\Gamma)$ for all $v, w \in \mathcal{H}$. 
The previous corollary immediately implies that the ergodicity or mixing of the $\Gamma$-action on $\Lambda \backslash H$ is equivalent to the ergodicity or mixing of the $\Gamma$-action on $T$ (see Corollary 6 below).

We apply Theorem 1 to the existence of a spectral gap for the random walk on $\Lambda \backslash H$ associated to a probability measure $\mu$ on $\operatorname{Aut}(\Lambda \backslash H)$.

Theorem 3 Let $\mu$ be a probability measure on $\operatorname{Aut}(\Lambda \backslash H)$. Denote by $\Gamma$ be the subgroup of Aut $(\Lambda \backslash H)$ generated by the support of $\mu$. Let $U^{0}$ and $V^{0}$ be the associated unitary representations of $\Gamma$ on $L_{0}^{2}(\Lambda \backslash H)$ and $L_{0}^{2}(T)$ respectively. Then

$$
\left\|U^{0}(\mu)\right\| \leq \max \left\{\left\|V^{0}(\mu)\right\|,\left\|\lambda_{\Gamma}(\mu)\right\|^{1 /(2 n+2)}\right\},
$$

where $\lambda_{\Gamma}$ is the left regular representation of $\Gamma$ on $\ell^{2}(\Gamma)$. In particular, the action of $\Gamma$ on $\Lambda \backslash H$ has a spectral gap if and only if the corresponding action of $\Gamma$ on $T$ has a spectral gap.

Example 4 Let $\Gamma$ be a subgroup of $\operatorname{Aut}(\Lambda \backslash H)$ such that its image $p(\Gamma) \subset$ $G L_{2 n}(\mathbf{Z})$ under the homomorphism $p: \operatorname{Aut}(\Lambda \backslash H) \rightarrow \operatorname{Aut}(T) \cong G L_{2 n}(\mathbf{Z})$ acts irreducibly on $\mathbf{R}^{2 n}$ and does not have an abelian subgroup of finite index (this is for instance the case if $p(\Gamma)$ is Zariski dense in $\left.G L_{2 n}(\mathbf{R})\right)$. Then, as shown in [FuSh99, Theorem 6.5], the action of $\Gamma$ on $T$ has a spectral gap.

In the case $n=1$, we have the following more precise result.

Corollary $\mathbf{5}$ Let $=H_{3}(\mathbf{R})$ be the 3 -dimensional Heisenberg group and $\Lambda$ a lattice in $H$. Let $\mu$ be a probability measure on $\operatorname{Aut}(\Lambda \backslash H)$. Then

$$
\left\|U^{0}(\mu)\right\| \leq\left\|\lambda_{\Gamma}(\mu)\right\|^{1 / 4},
$$

where $\Gamma$ is the subgroup generated by the support of $\mu$. In particular, if $\mu$ is aperiodic, $\left\|U^{0}(\mu)\right\|<1$ if and only if $\Gamma$ is non-amenable.

Acknowlegments We are grateful to J.-P. Conze, A. Gamburd and Y. Guivarc'h for interesting discussions. 


\section{Proofs}

We first recall the definition of the Heisenberg group $H=H_{2 n+1}(\mathbf{R})$; we then describe the automorphism group of $H$ as well as its irreducible unitary representations. by

Let $n \geq 1$ be an integer. Consider the symplectic form $\beta$ on $\mathbf{R}^{2 n}$ given

$$
\beta\left((x, y),\left(x^{\prime}, y^{\prime}\right)\right)=(x, y)^{t} J\left(x^{\prime}, y^{\prime}\right) \quad \text { for all } \quad(x, y),\left(x^{\prime}, y^{\prime}\right) \in \mathbf{R}^{2 n},
$$

where $J$ is the $(2 n \times 2 n)$-matrix

$$
J=\left(\begin{array}{cc}
0 & I_{n} \\
-I_{n} & 0
\end{array}\right)
$$

and $I_{n}$ is the $n \times n$-identity matrix. The $(2 n+1)$-dimensional Heisenberg group (over $\mathbf{R}$ ) is the group $H=H_{2 n+1}(\mathbf{R})$ with underlying set $\mathbf{R}^{2 n} \times \mathbf{R}$ and product

$$
((x, y), s)\left(\left(x^{\prime}, y^{\prime}\right), t\right)=\left(\left(x+x^{\prime}, y+y^{\prime}\right), s+t+\frac{1}{2} \beta\left((x, y),\left(x^{\prime}, y^{\prime}\right)\right)\right),
$$

for $(x, y),\left(x^{\prime}, y^{\prime}\right) \in \mathbf{R}^{2 n}, s, t \in \mathbf{R}$. This is a two-step nilpotent Lie group. Its centre $Z$ coincides with its commutator subgroup and is given by

$$
Z=\{((0,0), s): s \in \mathbf{R}\}
$$

The symplectic group $S p_{2 n}(\mathbf{R})$, which is the subgroup of $G L_{2 n}(\mathbf{R})$ of all matrices $g$ with ${ }^{t} g J g=J$, acts by automorphisms on $H=H_{2 n+1}(\mathbf{R})$ :

$$
g((x, y), t)=(g(x, y), t) \quad \text { for all } \quad g \in S p_{2 n}(\mathbf{R}),(x, y) \in \mathbf{R}^{2 n}, t \in \mathbf{R} .
$$

As is well-known (see [Foll89, 1.22]), the automorphism group $\operatorname{Aut}(H)$ of $H$ is generated by:

- the inner automorphisms,

- the automorphisms defined by matrices from $S p_{2 n}(\mathbf{R})$ as above,

- the dilations $((x, y), t) \mapsto\left((r x, r y), r^{2} t\right)$ for $r>0$, and

- the inversion $i:((x, y), t) \mapsto((y, x),-t)$. 
The connected component $\operatorname{Aut}(H)_{0}$ of the identity in $\operatorname{Aut}(H)$ is a subgroup of index two and can be viewed as the group of $(2 n+1) \times(2 n+1)$ matrices of the form

$$
\left(\begin{array}{cc}
r A & 0 \\
a^{t} & r^{2 n}
\end{array}\right)
$$

with $A \in S p_{2 n}(\mathbf{R}), r>0$, and $a$ a column vector in $\mathbf{R}^{2 n}$ (the action on $H$ corresponding to the usual action on $\mathbf{R}^{2 n+1}$ ). The subgroup of automorphisms of $H$ fixing pointwise the centre can be identified with the group of matrices of the form

$$
\left(\begin{array}{ll}
A & 0 \\
a^{t} & 1
\end{array}\right)
$$

and is hence isomorphic to the semi-direct product $S p_{2 n}(\mathbf{R}) \ltimes \mathbf{R}^{2 n}$, for the standard action of $S p_{2 n}(\mathbf{R})$ on $\mathbf{R}^{2 n}$.

The unitary dual $\widehat{H}$ of $H$ (that is, the set of classes of irreducible unitary representations of $H$ under unitary equivalence) consists of the equivalence classes of the following representations (see [Foll89, 1.50]):

- the unitary characters of the abelianized group $H / Z$;

- for every $t \in \mathbf{R} \backslash\{0\}$, the infinite dimensional representation $\pi_{t}$ defined on $L^{2}\left(\mathbf{R}^{n}\right)$ by the formula

$$
\pi_{t}((a, b), s) \xi(x)=\exp (2 \pi i t s) \exp \left(2 \pi\left\langle a, x-\frac{b}{2}\right\rangle\right) \xi(x-b)
$$

for $((a, b), s) \in H, \xi \in L^{2}\left(\mathbf{R}^{n}\right)$, and $x \in \mathbf{R}^{n}$.

For $t \neq 0$, the representation $\pi_{t}$ is, up to unitary equivalence, the unique irreducible unitary representation of $H$ whose restriction to the centre $Z$ is a multiple of the unitary character $s \mapsto \exp (2 \pi i t s)$.

The group $\operatorname{Aut}(H)$ acts on $\widehat{H}$ by

$$
\pi^{g}(h)=\pi\left(g^{-1}(h)\right) \quad \text { for all } \quad \pi \in \widehat{H}, g \in \operatorname{Aut}(H), h \in H .
$$

Let $g \in S p_{2 n}(\mathbf{R})$. For $t \in \mathbf{R} \backslash\{0\}$, the representation $\pi_{t}^{g}$ is unitary equivalent to $\pi_{t}$, since both representations have the same restriction to $Z$. Therefore, there exists a unitary operator $\sigma(g)$ on $L^{2}\left(\mathbf{R}^{n}\right)$ such that

$$
\sigma(g) \pi_{t}\left(g^{-1}(h)\right) \sigma(g)^{-1}=\pi_{t}(h) \quad \text { for all } \quad h \in H .
$$


By Schur's lemma, $\sigma(g)$ is unique up to a scalar multiple of the identity operator. Hence, for $g_{1}, g_{2} \in S p_{2 n}(\mathbf{R})$, there exists a complex number $c\left(g_{1}, g_{2}\right)$ of modulus one such that $\sigma\left(g_{1}\right) \sigma\left(g_{2}\right)=c\left(g_{1}, g_{2}\right) \sigma\left(g_{1} g_{2}\right)$. This means that $g \mapsto \sigma(g)$ is a projective unitary representation of $S p_{2 n}(\mathbf{R})$. We extend $\sigma$ to a projective unitary representation $\omega_{t}$, called Weil's representation, of $S p_{2 n}(\mathbf{R}) \ltimes \mathbf{R}^{2 n}$ by setting

$$
\omega_{t}(g, a)=\sigma(g) \pi_{t}(a) \quad \text { for all } \quad(g, a) \in S p_{2 n}(\mathbf{R}) \ltimes \mathbf{R}^{2 n} .
$$

Although we will not need this fact, it is worth mentioning that $\omega_{t}$ lifts to an ordinary representation of a two-fold cover of $S p_{2 n}(\mathbf{R}) \ltimes \mathbf{R}^{2 n}$ (see [Foll89, Chapter 4]).

How, let $\Lambda$ be a lattice in $H$. (As an example, $\Lambda$ can be the standard lattice $\left\{((x, y), s / 2): x, y \in \mathbf{Z}^{n}, s \in \mathbf{Z}\right\}$; a full classification of the lattices in $H$ is given in [Ausl77, I. 2].)

The Lebesgue measure on $\mathbf{R}^{2 n} \times \mathbf{R}$ is a Haar measure on $H$ and induces an invariant measure $m$ on the nilmanifold $\Lambda \backslash H$. (For the classification of $\Gamma$-invariant measures on $\Lambda \backslash H$ for "large" groups $\Gamma \subset \operatorname{Aut}(\Lambda \backslash H)$, see [Heu09].)

\section{Proof of Theorem 1.}

Let $\Gamma$ be a subgroup of $\operatorname{Aut}(\Lambda \backslash H)$. Then $\Gamma$ is a discrete subgroup of $\operatorname{Aut}(H)$, for the topology of uniform convergence on compact subsets of $H$. Moreover, the subgroup of $\Gamma$ consisting of the automorphisms fixing pointwise the centre of $H$ has finite index in $\Gamma$. Indeed, the mapping

$$
\operatorname{Aut}(H)_{0} \rightarrow \mathbf{R}^{*}, \quad\left(\begin{array}{cc}
r A & 0 \\
a^{t} & r^{2 n}
\end{array}\right) \mapsto r
$$

is a homomorphism and the image of $\Gamma \cap \operatorname{Aut}(H)_{0}$ is a discrete subgroup of $\mathbf{R}^{*}$.

It is clear that, if Theorem 1 is true for a subgroup of finite index in $\Gamma$, then it is true for $\Gamma$. So, we can (and will) assume that $\Gamma$ is a subgroup of $S p_{2 n}(\mathbf{R}) \ltimes \mathbf{R}^{2 n}$.

Since every $\gamma \in \Gamma$ preserves the measure $m$ on $\Lambda \backslash H$, we have an associated unitary representation $U: \gamma \mapsto U_{\gamma}$ of $\Gamma$ on $L^{2}(\Lambda \backslash H, m)$.

Let $\rho_{\Lambda \backslash H}$ be the unitary representation of $H$ on $L^{2}(\Lambda \backslash H, m)$ given by right translation:

$$
\rho_{\Lambda \backslash H}(h) \xi(x)=\xi(x h) \quad \text { for all } \quad h \in H, \xi \in L^{2}(\Lambda \backslash H, m), x \in \Lambda \backslash H .
$$


The representations $U$ and $\rho_{\Lambda \backslash H}$ are linked in the following way. For every $\gamma \in \Gamma$, we have:

$$
U_{\gamma} \rho_{\Lambda \backslash H}(h) U_{\gamma^{-1}}=\rho_{\Lambda \backslash H}(\gamma(h)) \quad \text { for all } \quad h \in H .
$$

We have a decomposition of $L^{2}(\Lambda \backslash H, m)$ into $\rho_{\Lambda \backslash H^{-}}$-invariant subspaces

$$
L^{2}(\Lambda \backslash H, m)=\bigoplus_{m \in \mathbf{Z}} \mathcal{H}_{m}
$$

where

$$
\mathcal{H}_{m}=\left\{\xi \in L^{2}(\Lambda \backslash H): \rho_{\Lambda \backslash H}(0,0, s) \xi=e^{2 \pi i m s} \xi \quad \text { for all } \quad t \in \mathbf{R}\right\} .
$$

The space $\mathcal{H}_{0}$ coincides with the space $L^{2}(T)$, where $T \cong \mathbf{R}^{2 n} / \mathbf{Z}^{2 n}$ is the maximal torus factor associated to $\Lambda \backslash H$. Moreover, for every $m \in \mathbf{Z} \backslash\{0\}$, the subspace $\mathcal{H}_{m}$ is an isotypical component for $\rho_{\Lambda \backslash H}$ and is equivalent to a finite multiple of the irreducible representation $\pi_{m}$ from above. (For a computation of the multiplicities, see [Toli78], [Moor65].)

Let $m \in \mathbf{Z} \backslash\{0\}$. Since $\Gamma$ fixes pointwise $Z$, we see from (1) that

$$
U_{\gamma}\left(\mathcal{H}_{m}\right)=\mathcal{H}_{m} \quad \text { for all } \quad \gamma \in \Gamma .
$$

Denote by $U^{(m)}$ the restriction of $U$ to $\mathcal{H}_{m}$.

Since $\mathcal{H}_{m}$ is equivalent to a finite multiple of the irreducible representation $\pi_{m}$, we can assume that $\mathcal{H}_{m}$ is the tensor product

$$
\mathcal{H}_{m}=\mathcal{K}_{m} \otimes \mathcal{L}_{m}
$$

of the Hilbert space $\mathcal{K}_{m}$ of $\pi_{m}$ with a finite dimensional Hilbert space $\mathcal{L}_{m}$, in such a way that

$$
\left.\rho_{\Lambda \backslash H}(h)\right|_{\mathcal{H}_{m}}=\pi_{m}(h) \otimes I_{\mathcal{L}_{m}} \quad \text { for all } \quad h \in H .
$$

Let $\gamma \in \Gamma$. By (1) and (2) above, we have

$$
U_{\gamma}^{(m)}\left(\pi_{m}(h) \otimes I_{\mathcal{L}_{m}}\right) U_{\gamma^{-1}}^{(m)}=\pi_{m}(\gamma(h)) \otimes I_{\mathcal{L}_{m}} \quad \text { for all } \quad x \in H .
$$

Let $\omega_{m}$ be Weil's representation from above. Recall that $\omega_{m}$ is a projective representation of $S p_{2 n}(\mathbf{R}) \ltimes \mathbf{R}^{2 n}$ defined on $\mathcal{K}_{m}=L^{2}\left(\mathbf{R}^{n}\right)$ and that $\omega_{m}$ extends $\pi_{m}$. We have

$$
\omega_{m}(\gamma) \pi_{m}(h) \omega_{m}(\gamma)^{-1}=\pi_{m}(\gamma(h)) \quad \text { for all } \quad h \in H .
$$


It follows from (3) and (4) that, on $\mathcal{H}_{m}$, the operator $\left(\omega_{m}(\gamma)^{-1} \otimes I_{\mathcal{L}_{m}}\right) U_{\gamma}^{(m)}$ commutes with $\left(\pi_{m}(h) \otimes I_{\mathcal{L}_{m}}\right)$ for all $h \in H$. Hence, since $\pi_{m}$ is irreducible, there exists a bounded operator $V_{\gamma}^{(m)}$ on $\mathcal{L}_{m}$ such that

$$
\left(\omega_{m}(\gamma)^{-1} \otimes I_{\mathcal{L}_{m}}\right) U_{\gamma}^{(m)}=I_{\mathcal{K}_{m}} \otimes V_{\gamma}^{(m)}
$$

that is,

$$
U_{\gamma}^{(m)}=\omega_{m}(\gamma) \otimes V_{\gamma}^{(m)}
$$

Since $U^{(m)}$ is a unitary representation, it is clear that $\gamma \mapsto V_{\gamma}^{(m)}$ is a projective unitary representation of $\Gamma$.

Let $\xi, \eta \in \mathcal{S}\left(\mathbf{R}^{n}\right)$ be Schwartz functions on $\mathbf{R}^{n}$. By [HoMo79, Proposition 6.4], for every $\varepsilon>0$, the matrix coefficient

$$
C_{\xi, \eta}^{\omega_{m}}: g \mapsto\left\langle\omega_{m}(g) \xi, \eta\right\rangle
$$

of the metaplectic representation $\omega_{m}$ belongs to $L^{4 n+2+\varepsilon}\left(S p_{2 n}(\mathbf{R}) \ltimes \mathbf{R}^{2 n}\right)$. Set $G=S p_{2 n}(\mathbf{R}) \ltimes \mathbf{R}^{2 n}$; observe that $\Gamma$ is a discrete and hence closed subgroup of $G$. Choosing a Borel subset $X \subset G$ which is a fundamental domain for the quotient space $\Gamma \backslash G$, we can write (compare with the proof of Proposition 6.4 in [Howe82])

$$
\begin{aligned}
\int_{G}\left|C_{\xi, \eta}^{\omega_{m}}(g)\right|^{4 n+2+\varepsilon} d g & \left.=\int_{G} \mid\left\langle\omega_{m}(g) \xi, \eta\right\rangle\right)\left.\right|^{4 n+2+\varepsilon} d g \\
& =\int_{X}\left(\sum_{\gamma \in \Gamma}\left|\left\langle\omega_{m}(\gamma g) \xi, \eta\right\rangle\right|^{4 n+2+\varepsilon}\right) d g \\
& <\infty
\end{aligned}
$$

Therefore, by Fubini's theorem, for almost every $g \in X$, we have

$$
\sum_{\gamma \in \Gamma}\left|\left\langle\omega_{m}(\gamma) \omega_{m}(g) \xi, \eta\right\rangle\right|^{4 n+2+\varepsilon}<\infty
$$

that is, $C_{\omega_{m}(g) \xi, \eta}^{\left.\omega_{m}\right|_{\Gamma}} \in \ell^{4 n+2+\varepsilon}(\Gamma)$.

Since $\mathcal{S}\left(\mathbf{R}^{n}\right)$ contains a countable set which is dense in $L^{2}\left(\mathbf{R}^{n}\right)$, il follows that there exist dense subspaces $D_{1}^{(m)}$ and $D_{2}^{(m)}$ of $L^{2}\left(\mathbf{R}^{n}\right)$ such that

$$
C_{\xi, \eta}^{\left.\omega_{m}\right|_{\Gamma}} \in \ell^{4 n+2+\varepsilon}(\Gamma)
$$


for all $\xi \in D_{1}^{(m)}$ and $\eta \in D_{2}^{(m)}$.

Since $U_{\gamma}^{(m)}=\omega_{m}(\gamma) \otimes V_{\gamma}^{(m)}$ and since matrix coefficients of projective unitary representations are bounded, the matrix coefficients $C_{\xi \otimes \xi^{\prime}, \eta \otimes \eta^{\prime}}^{U^{(m)}}$ of $U^{(m)}$ belong to $\ell^{4 n+2+\varepsilon}(\Gamma)$ for $\xi \in D_{1}^{(m)}, \eta \in D_{2}^{(m)}$ and $\xi^{\prime} \in \mathcal{L}_{m}, \eta^{\prime} \in \mathcal{L}_{m}$.

Let now $D_{1}, D_{2}$ be the linear subspaces of $\mathcal{H}$ generated respectively by

$$
\left\{\xi \otimes \xi^{\prime}: \xi \in D_{1}^{(m)}, \xi^{\prime} \in \mathcal{L}_{m}, m \in \mathbf{Z} \backslash\{0\}\right\}
$$

and

$$
\left\{\eta \otimes \eta^{\prime}: \eta \in D_{2}^{(m)}, \eta^{\prime} \in \mathcal{L}_{m}, m \in \mathbf{Z} \backslash\{0\}\right\} .
$$

Then $D_{1}$ and $D_{2}$ are dense in $\mathcal{H}$ and the matrix coefficients $C_{v, w}^{U}$ belong to $\ell^{4 n+2+\varepsilon}(\Gamma)$ for $v \in D_{1}$ and $w \in D_{2}$.

\section{Proof of Theorem 3}

Let $\mu$ be a probability measure on $\operatorname{Aut}(\Lambda \backslash H)$. Denote by $\Gamma$ the subgroup of $\operatorname{Aut}(\Lambda \backslash H)$ generated by the support of $\mu$.

Let $\lambda_{\Gamma}$ be the left regular representation of $\Gamma$ on $\ell^{2}(\Gamma)$. Let $U^{0}$ and $V^{0}$ be the corresponding unitary representations of $\Gamma$ on $L_{0}^{2}(\Lambda \backslash H)$ and $L_{0}^{2}(T)$, respectively. We claim that

$$
\left\|U^{0}(\mu)\right\| \leq \max \left\{\left\|V^{0}(\mu)\right\|,\left\|\lambda_{\Gamma}(\mu)\right\|^{1 / k}\right\}
$$

for $k=2 n+2$.

Denoting by $U^{\mathcal{H}}$ the restriction of $U$ to $\mathcal{H}$, it suffices to show that

$$
\left\|U^{\mathcal{H}}(\mu)\right\| \leq\left\|\lambda_{\Gamma}(\mu)\right\|^{1 / k} .
$$

By Theorem 1, the matrix coefficients $C_{v, w}^{U^{\mathcal{H}}}$ of $U^{\mathcal{H}}$ are in $\ell^{2 k}(\Gamma)$ for $v$ and $w$ in dense subspaces $D_{1}$ and $D_{2}$ of $\mathcal{H}$. It follows that the $k$-fold tensor power $\left(U^{\mathcal{H}}\right)^{\otimes k}$ of $U^{\mathcal{H}}$ is unitarily equivalent to a subrepresentation of an infinite multiple $\infty \lambda_{\Gamma}$ of $\lambda_{\Gamma}$ (see [HoTa92, Chapter V, 1.2.4]). Hence,

$$
\left\|\left(U^{\mathcal{H}}\right)^{\otimes k}(\mu)\right\| \leq\left\|\infty \lambda_{\Gamma}(\mu)\right\|=\left\|\lambda_{\Gamma}(\mu)\right\|
$$

and Inequality (7) will be proved if we show that

$$
\left\|U^{\mathcal{H}}(\mu)\right\| \leq\left\|\left(U^{\mathcal{H}}\right)^{\otimes k}(\mu)\right\|^{1 / k}
$$


To show Inequality (8), we use the following argument from [Nevo98]. Denote by $\check{\mu}$ the probability measure on $\Gamma$ defined by $\check{\mu}(\gamma)=\mu\left(\gamma^{-1}\right)$. For every vector $v \in \mathcal{H}$, using Jensen's inequality, we have

$$
\begin{aligned}
\|U(\mu) v\|^{2 k} & =|\langle U(\check{\mu} * \mu) v, v\rangle|^{k} \\
& =\left|\sum_{\gamma \in \Gamma}\langle U(\gamma) v, v\rangle(\check{\mu} * \mu)(\gamma)\right|^{k} \\
& \leq \sum_{\gamma \in \Gamma}|\langle U(\gamma) v, v\rangle|^{k}(\check{\mu} * \mu)(\gamma) \\
& =\sum_{\gamma \in \Gamma}\left\langle U(\gamma)^{\otimes k} v^{\otimes k}, v^{\otimes k}\right\rangle(\check{\mu} * \mu)(\gamma) \\
& =\left|\left\langle U^{\otimes k}(\check{\mu} * \mu) v^{\otimes k}, v^{\otimes k}\right\rangle\right| \\
& =\left\|U^{\otimes k}(\mu) v^{\otimes k}\right\|^{2} .
\end{aligned}
$$

Hence, $\left\|U^{\mathcal{H}}(\mu)\right\| \leq\left\|\left(U^{\mathcal{H}}\right)^{\otimes k}(\mu)\right\|^{1 / k}$, as claimed.

Assume that $\mu$ is aperiodic and that the $\Gamma$ action on $T$ has a spectral gap. Then, as mentioned in the Introduction, $\Gamma$ is not amenable. Hence, $\left\|\lambda_{\Gamma}(\mu)\right\|<1$ (see [BeHV08, G.4.2]) and therefore $\left\|U^{0}(\mu)\right\|<1$, that is, the $\Gamma$ action on $\Lambda \backslash H$ has a spectral gap.

\section{Proof of Corollary 5}

Let $H=H_{3}(\mathbf{R})$ be the 3-dimensional Heisenberg group and $\Lambda$ a lattice in $H$. The unitary representation $V$ of $\operatorname{Aut}(\Lambda \backslash H)$ on $L^{2}(T)$ factors through $p$ : $\operatorname{Aut}(\Lambda \backslash H) \rightarrow \operatorname{Aut}(T) \cong G L_{2}(\mathbf{Z})$ to the standard representation of $G L_{2}(\mathbf{Z})$ on $L^{2}(T)=L^{2}\left(\mathbf{R}^{2} / \mathbf{Z}^{2}\right)$. By Fourier duality, this last representation is unitarily equivalent to the representation of $G L_{2}(\mathbf{Z})$ on $\ell^{2}\left(\mathbf{Z}^{2}\right)$ obtained from the dual action of $G L_{2}(\mathbf{Z})$ on $\mathbf{Z}^{2}$. We have an orthogonal decomposition into $G L_{2}(\mathbf{Z})$ invariant subspaces

$$
\ell^{2}\left(\mathbf{Z}^{2}\right)=\mathbf{C} \delta_{0} \bigoplus \bigoplus_{t \in T} \ell^{2}\left(G L_{2}(\mathbf{Z}) / \Gamma_{t}\right)
$$

where $T$ is a set of representatives for the $G L_{2}(\mathbf{Z})$-orbits in $\mathbf{Z}^{2} \backslash\{0\}$ and $\Gamma_{t}$ is the stabilizer of $t$ in $G L_{2}(\mathbf{Z})$. Since every $\Gamma_{t}$ is solvable (and hence amenable), it follows that $V^{0}$ is weakly contained in $\lambda_{G L_{2}(\mathbf{Z})} \circ p$ (see [BeHV08, Appendix F]). Hence, for every probability measure $\mu$ on Aut $(\Lambda \backslash H)$, we have

$$
\left\|V^{0}(\mu)\right\| \leq\left\|\left(\lambda_{G L_{2}(\mathbf{Z})} \circ p\right)(\mu)\right\| .
$$


Since

$$
\operatorname{ker} p \subset\left\{\left(\begin{array}{ll}
I_{2} & 0 \\
a^{t} & 1
\end{array}\right): a \in \mathbf{R}^{2}\right\} \cong \mathbf{R}^{2},
$$

ker $p$ is amenable and it follows that

$$
\left\|\left(\lambda_{G L_{2}(\mathbf{Z})} \circ p\right)(\mu)\right\| \leq\left\|\lambda_{\operatorname{Aut}(\Lambda \backslash H)}(\mu)\right\| .
$$

Therefore, we have

$$
\left\|V^{0}(\mu)\right\| \leq\left\|\lambda_{\operatorname{Aut}(\Lambda \backslash H)}(\mu)\right\| .
$$

Denote by $\Gamma$ the subgroup generated by the support of $\mu$. Since

$$
\left\|\lambda_{\Gamma}(\mu)\right\|=\left\|\lambda_{\operatorname{Aut}(\Lambda \backslash H)}(\mu)\right\|
$$

it follows from Theorem 3 that

$$
\left\|U^{0}(\mu)\right\| \leq \max \left\{\left\|V^{0}(\mu)\right\|,\left\|\lambda_{\Gamma}(\mu)\right\|^{1 / 4}\right\}=\left\|\lambda_{\Gamma}(\mu)\right\|^{1 / 4} .
$$

Assume that $\mu$ is aperiodic. If $\Gamma$ is non-amenable, then $\left\|\lambda_{\Gamma}(\mu)\right\|<1$ and hence $\left\|U^{0}(\mu)\right\|<1$. If $\Gamma$ is amenable, then $\Gamma$ has no spectral gap in $\Lambda \backslash H$.

\section{Some further applications}

Let $G$ be a locally compact group acting by measure preserving transformations on a probability space $(X, m)$. Let $U$ denote the associated unitary representation of $G$ on $L^{2}(X, m)$. The action of $G$ on $X$ is weakly mixing if $L_{0}^{2}(X, m)$ contains no non-zero finite dimensional $U(G)$-invariant subspace (equivalently: if the diagonal action of $G$ on $X \times X$ is ergodic; see [BeMa00, Chapter I, 2.17]). The action is strongly mixing if, for all $\xi, \eta \in L_{0}^{2}(X, m)$, the matrix coefficient $g \mapsto\left\langle U_{g} \xi, \eta\right\rangle$ belongs to $C_{0}(G)$.

With the notation of Theorem 1 , all matrix coefficients $C_{v, w}^{U}$ are in $c_{0}(\Gamma)$ for $v \in D_{1}$ and $w \in D_{2}$. By density of $D_{1}$ and $D_{2}$ in $\mathcal{H}$, the same is true for all $v, w \in \mathcal{H}$. It follows that $\mathcal{H}$ contains no non-zero finite dimensional $U(G)$-invariant subspace if $\Gamma$ is infinite (see [BeMa00, Chapter I, 2.15.iii]). Therefore, we immediately obtain the following corollary.

Corollary 6 Let $\Gamma$ be a group of automorphisms of the compact Heisenberg nilmanilfold $\Lambda \backslash H$ and $T$ the maximal $T$ torus factor associated to $\Lambda \backslash H$. The following properties are equivalent. 
(i) The action of $\Gamma$ on $\Lambda \backslash H$ is ergodic (weakly mixing or strongly mixing, respectively).

(ii) The action of $\Gamma$ on $T$ is ergodic (weakly mixing or strongly mixing, respectively)

Remark 7 In the case where $\Gamma$ is generated by a single automorphism (or even an affine transformation) of an arbitrary compact nilmanifold, the previous corollary was obtained by W. Parry (see [Parr69], [Parr70]). The result concerning ergodicity has been generalized by J.-P. Conze ([Conz09]) to arbitrary groups of affine transformations of a general compact nilmanifold $\Lambda \backslash H$. Moreover, [Conz09] gives an example of an ergodic group $\Gamma$ of automorphisms of the standard 7-dimensional Heisenberg nilmanifold $\Lambda \backslash H_{7}(\mathbf{R})$ such that no element $\gamma \in \Gamma$ acts ergodically on $\Lambda \backslash H_{7}(\mathbf{R})$.

Let $G$ be a locally compact group acting by measure preserving transformations on a probability space $(X, m)$ and $U$ the associated unitary representation of $G$ on $L^{2}(X, m)$. Assume that $\left\|\left(U^{0} \otimes U^{0}\right)(\mu)\right\|<1$. This condition, which is formely stronger than the spectral gap condition $\left\|U^{0}(\mu)\right\|<1$, plays an important role in [FuSh99]. Indeed, it is shown in Theorem 1.4 there that, with the notation as in the Introduction, for every $\xi, \eta \in L^{2}(X, m)$, the correlation coefficient $\left\langle U\left(S_{n}^{\omega}\right) \xi, \eta\right\rangle$ converge almost surely to $\int_{X} \xi d m \int_{X} \eta d m$, with exponentially fast speed.

However, we can see that both conditions are equivalent in our situation.

Corollary 8 With the notation as in Theorem 3, the following properties are equivalent.

(i) $\left\|\left(U^{0} \otimes U^{0}\right)(\mu)\right\|<1$;

(ii) $\left\|U^{0}(\mu)\right\|<1$;

(iii) $\left\|V^{0}(\mu)\right\|<1$.

Indeed, a proof similar to the one of Theorem 3 shows that the following inequality holds:

$$
\left\|\left(U^{0} \otimes U^{0}\right)(\mu)\right\| \leq \max \left\{\left\|\left(V^{0} \otimes V^{0}\right)(\mu)\right\|,\left\|\lambda_{\Gamma}(\mu)\right\|^{1 / k}\right\} .
$$

On the other hand, as was shown in [FuSh99, Theorem 6.4], the condition $\left\|V^{0}(\mu)\right\|<1$ is equivalent to the condition $\left\|\left(V^{0} \otimes V^{0}\right)(\mu)\right\|<1$. This shows the equivalence of Conditions (i), (ii), and (iii). 


\section{References}

[Ausl77] L. Auslander. Lecture notes on nil-theta functions, CBMS Regional Conference Series 34, American Math. Soc. 1977

[BeHV08] B. Bekka, P. de la Harpe, A. Valette. Kazhdan's Property (T), Cambridge University Press 2008.

[BeMa00] B. Bekka, M. Mayer. Ergodic theory and topological dynamics of group actions on homogeneous spaces, London Math. Soc. Lecture Note Series 269, Cambridge University Press 2000.

[Conz09] J.-P. Conze. Action d'un groupe d'applications affines sur une nilvariété compacte. Preprint 2009

[Foll89] G.B. Folland. Harmonic analysis in phase space, Annals of Mathematics Studies 122, Princeton University Press 1989.

[FuSh99] A. Furman, Y. Shalom. Sharp ergodic theorems for group actions and strong ergodicity. Ergod. Th. \& Dynam. Sys. 19, 1037-1061 (1999).

[Guiv05] Y. Guivarc'h. Limit theorems for random walks and products of random matrices. In:Probability measures on groups: recent directions and trends, 255-330, Tata Inst. Fund. Res., Mumbai, 2006.

[Heu09] J-R. Heu. Dynamical properties of groups of automorphisms on Heisenberg nilmanifolds. Geometriae Dedicata, to appear.

[Howe82] R. Howe. On a notion of rank for unitary representations of the classical groups. In:Harmonic analysis and group representations. 223-331, Liguori, Haples, 1982.

[HoMo79] R. Howe, C. C. Moore. Asymptotic properties of unitary representations. J. Funct. Anal. 32, 72-96 (1979).

[HoTa92] R. Howe, E.C. Tan. Hon-abelian harmonic analysis, Springer 1992.

[JuRo79] A. del Junco, J. Rosenblatt. Counterexamples in ergodic theory and number theory. Math. Ann. 245, 185-197 (1979). 
[Lubo94] A. Lubotzky. Discrete groups, expanding graphs and invariant measures, Birkhäuser 1994.

[Moor65] C. C. Moore. Decomposition of unitary representations defined by discrete subgroups of nilpotent Lie groups. Ann. Math. 82, 146-182 (1965).

[Nevo98] A. Nevo. Spectral transfer and pointwise ergodic theorems for semisimple Kazhdan groups, Math. Res.Letters 5, 305-325, 1998.

[Parr69] W. Parry. Ergodic properties of affine transformations and flows on nilmanifolds. Amer. J. Math.9, 757-771 (1969).

[Parr70] W. Parry. Dynamical systems on nilmanifolds. Bull. London Math. Soc. 2, 37-40 (1970).

[Sarn90] P. Sarnak. Some applications of modular forms, Cambridge University Press, 1990.

[Schmi80] K. Schmidt. Asymptotically invariant sequences and an action of $S L(2, \mathbf{Z})$ on the 2-sphere. Israel J. Math. 37, 193-208 (1980).

[Shal62] D. Shale. Linear symmetries of free boson fields. Trans. Amer. Math. Soc. 103, 149-167 (1962).

[Toli78] R. Tolimieri. Heisenberg manifolds and theta functions. Trans. Amer. Math. Soc. 239, 293-319 (1978).

[Weil64] A. Weil. Sur certains groupes d'opérateurs unitaires. Acta Math. 111, 143-211 (1964).

\section{Address}

UFR Mathématiques, Université de Rennes 1,

Campus Beaulieu, F-35042 Rennes Cedex

France

E-mail : bachir.bekka@univ-rennes1.fr, jean-romain.heu@univ-rennes1.fr 